Check for updates

Cite this: RSC Adv., 2019, 9, 17537

Received 29th April 2019

Accepted 17th May 2019

DOI: 10.1039/c9ra03182k

rsc.li/rsc-advances

\section{Controlling upconversion luminescence patterns in space with red emission enhancement from a single fluoride microcrystal by tuning the excitation mode}

\author{
Qingyan Han, (D) *a Zhu Lu, ${ }^{\text {a }}$ Wei Gao, ${ }^{a}$ Wanting Zhou, ${ }^{\mathrm{a}}$ Jianxia Qi, ${ }^{\mathrm{b}}$ Aihua Hao ${ }^{\mathrm{a}}$ \\ and Jun Dong*a
}

The ability to control the upconversion (UC) luminescence patterns in space from lanthanide-doped UC luminescence materials is very important for many applications including three-dimensional color displays, optical waveguides and optical communication. In this work, the fascinating UC luminescence patterns could be adjusted from the blue or green flower-like emission pattern to the red flame-like irradiation pattern with a red luminescence enhancement from a single $\beta-\mathrm{NaYF}_{4}: \mathrm{Yb}^{3+} /\left(\mathrm{Tm}^{3+}\right.$ or $\left.\mathrm{Er}^{3+}\right)$ microcrystal $(M C)$ by varying the excitation position. The red-to-blue $(R / B)$ and the red-to-green $(R / G)$ emission intensity ratios from the single $M C$ particle that the focal point position of excitation NIR laser is on the side lace are much stronger than that in the case of the focused laser beam on the center. We think that the physical mechanism from the changes in the luminescence pattern and the emission intensity ratio is explained by the optical waveguide effect based on the total reflection effect. These results provide a new strategy for facilitating fundamental investigations of the UC micro/nano-materials, which will lead to promising applications in three-dimensional color display, optical waveguides and optical communication.

\section{Introduction}

Upconversion (UC) luminescence is a process that can convert near-infrared (NIR) excitation into visible and ultraviolet emission. ${ }^{\mathbf{1 , 2}}$ It shows photophysical properties such as high signal to noise ratio, long luminescence lifetime, sharp emission bands, excellent photostability, high photochemical stability, and low toxicity. $^{3-6}$ Based on these advantages, lanthanide-doped UC luminescence materials have gained much attention in fundamental research and various frontier applications including photoinduced therapy, temperature sensing, solid-state lasers, photovoltaic conversion, biological imaging, and 3D displays. $^{7-15}$ Although the morphology, crystal structure, and emission colors of UC luminescence materials could be accurately controlled, the high-efficiency UC emission and luminescence color or intensity manipulation of lanthanide-doped UC materials can't meet some specific applications in bioimaging and therapy. ${ }^{\mathbf{1 6 , 1 7}}$ Searching for more efficient host materials, designing core-shell structures to alleviate surfacerelated quenching, and using surface plasmon resonance to enhance the local optical field is still explore to date. ${ }^{18-22}$

${ }^{a}$ School of Electronic Engineering, Xi'an University of Posts and Telecommunications, Xi'an 710121, China.E-mail: qyhan@xupt.edu.cn; dongjun@xupt.edu.cn

${ }^{b}$ School of Science, Xi'an University of Posts and Telecommunications, Xi'an 710121, China
As is well known, fluoride matrix is usually regarded as much more effective luminescence host materials due to the advantages of low energy phonons and transparency. ${ }^{23}$ Many micro/ nano-materials with various morphologies and structures have been synthesized, including rods, prism, tubes, octahedron and some recently reports about hexagonal-phase $\mathrm{NaYbF}_{4}$ microrod with one-dimensional hollow microstructure, $\mathrm{NaErF}_{4} @ \mathrm{NaYF}_{4}$ core-shell nanocrystals, and $\mathrm{Yb}^{3+}-\mathrm{Er}^{3+}-\mathrm{Tm}^{3+}$ tridoped hexagonal $\beta-\mathrm{NaYF}_{4}$ microrod. ${ }^{24-29}$ Among these lanthanide-doped fluoride materials, the brighter UC emission light could be achieved through searching for good configuration of the particle morphology, controlling optimum composition and concentration of lanthanide dopant, and introducing suitable noble metal-nanostructures. However, it is rarely reported on modulation of the UC luminescence intensity and patterns in space. The UC luminescence based on the waveguide effect may overcome these issues, in which the tight confinement of excitation light could not only be propagated along certain direction of in the media, but also effectively enhanced luminescence intensity by guiding light with increasing the optical path. ${ }^{30-32}$ Hence, this an opportunity to achieve UC luminescence patterns manipulation and irradiation intensity enhancement by controlling the excitation conditions.

In this work, two interesting UC luminescence patterns including flower-shaped emission and red flame-like irradiation from a single $\beta-\mathrm{NaYF}_{4}: \mathrm{Yb}^{3+} /\left(\mathrm{Tm}^{3+}\right.$ or $\left.\mathrm{Er}^{3+}\right) \mathrm{MC}$ with special 
structure are obtained via varying the excitation NIR laser position. In addition, the corresponding emission bands intensity would be also redistributed in space that results in a red emission enhancement with changing the excitation position based on the optical waveguide effect. The luminescence mechanism of the spatial control of the emission pattern and intensity were deeply investigated.

\section{Experimental}

\subsection{Sample preparation}

In all experimental processes, all the chemicals were used without further purification. The lanthanide oxides $\left(\operatorname{Ln}_{2} \mathrm{O}_{3}\right.$ (99.99\%), Ln = Y, Yb, Er, Tm) were supplied by Sigma-Aldrich. Sodium Fluoride (NaF, 98.00\%) and sodium citrate $\left(\mathrm{Na}_{3} \mathrm{C}_{6} \mathrm{H}_{5}{ }^{-}\right.$ $\mathrm{O}_{7} \cdot 2 \mathrm{H}_{2} \mathrm{O}, 99.00 \%$ ) were purchased from Sinopharm Chemical Reagent Co., Ltd. of China. Ultrapure deionized water was used throughout the experiments.

The $\beta-\mathrm{NaYF}_{4}: \mathrm{Yb}^{3+}-\mathrm{Er}^{3+} / \mathrm{Tm}^{3+}$ microcrystals (MCs) were synthesized by a modified hydrothermal method. ${ }^{33}$ In a typical process, the $\mathrm{Ln}_{2} \mathrm{O}_{3}(\mathrm{Ln}=\mathrm{Y}, \mathrm{Yb}, \mathrm{Er}$, and $\mathrm{Tm})$ powders were dissolved in dilute nitric acid, respectively. The solution is stirred at $60{ }^{\circ} \mathrm{C}$ to evaporate the superfluous nitric acid. A colorless powder was dissolved in deionized water to form $\mathrm{Ln}\left(\mathrm{NO}_{3}\right)_{3}(\mathrm{Ln}=\mathrm{Y}, \mathrm{Yb}, \mathrm{Er}$, and $\mathrm{Tm})$ solutions. $0.3 \mathrm{mmol}$ of $\mathrm{Ln}\left(\mathrm{NO}_{3}\right)_{3}(\mathrm{RE}=\mathrm{Y}, \mathrm{Yb}$, and $\mathrm{Tm})$ aqueous solutions were added into $21.0 \mathrm{ml}$ of deionized water and $0.5 \mathrm{mmol}$ of sodium citrate. The mixture was magnetically stirred for $30 \mathrm{~min}$ at room temperature. Then, $6.0 \mathrm{ml}$ of $\mathrm{NaF}(1.0 \mathrm{M})$ aqueous solution was added into the above solution, and the obtained solution was vigorously stirred for $25 \mathrm{~min}$. Subsequently, the suspension was transferred to a Teflon-lined autoclave, and heated at $200{ }^{\circ} \mathrm{C}$ for $24 \mathrm{~h}$. The resultant product was centrifugated at $4000 \mathrm{rpm}$ for $5 \mathrm{~min}$, washed with deionized water and ethanol three times, and dried under $60{ }^{\circ} \mathrm{C}$ for several hours.

\subsection{Measurements}

The morphology study of the as-prepared sample was characterized by using a scanning electron microscope (SEM, FEI-Nova NanoSEM 450). The phase purity of the samples was obtained with a Rigaku D/Max2550VB+/PC X-ray diffractometer with a graphite monochromatic $\mathrm{Cu}-\mathrm{K} \alpha$ radiation source $(\lambda=0.15406$ $\mathrm{nm})$. The UC luminescence property of a single MC was investigated by a confocal microscopy system. The UC luminescence spectra were recorded and analyzed by a spectrometer (SP2750i, $0.008 \mathrm{~nm}$ ) equipped with a PIXIS 100 charge coupled device (CCD, ACTON) and a PD471 photomultiplier tube (PMT, ACTON). The wavelength of the excitation light was $980 \mathrm{~nm}$. All measurements were performed at room temperature.

\section{Results and discussion}

Hexagonal $\mathrm{NaYF}_{4}$ MCs were successfully prepared by a facile hydrothermal approach with the assistance of trisodium citrate. Fig. 1a shows the scanning electron microscope (SEM) image of $\mathrm{NaYF}_{4} \mathrm{MCs}$, which implies the purity and uniformity of the

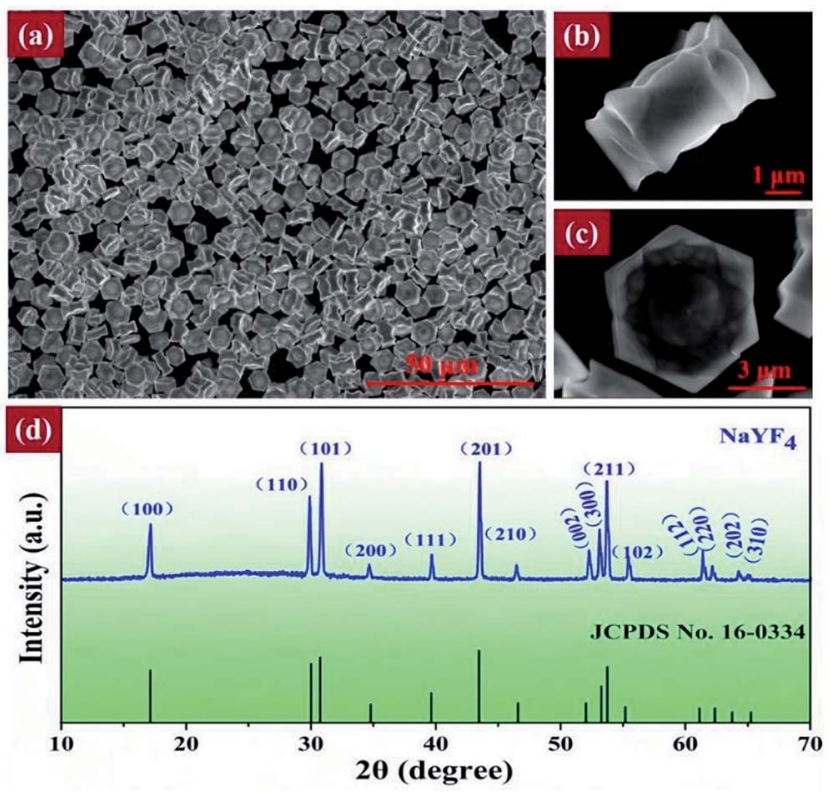

Fig. 1 (a) SEM image of the as-synthesized $\mathrm{NaYF}_{4}$ hexagonal MCs. (bc) SEM images of the single MC. (d) XRD pattern of the MCs and literature data for hexagonal phase $\mathrm{NaYF}_{4}$ (JCPDS file no. 16-0334).

products. The size of MC particles has an average dimension of $\approx 5.0 \mu \mathrm{m}$ and thicknesses of $\approx 2.0 \mu \mathrm{m}$. Intriguingly, the $\mathrm{NaYF}_{4}$ MC consists of an arc-shaped hexagonal plate and six petalshaped side walls (Fig. 1b and c). The X-ray diffraction (XRD) spectrum provides an evidence that the MCs are pure hexagonal $\mathrm{NaYF}_{4}$ phase (JCPDS file no. 16-0334) as shown in Fig. 1d. It should be mentioned that there is no variation in the crystal structure of $\mathrm{NaYF}_{4}$ when $\mathrm{Yb}^{3+}$ and $\mathrm{RE}^{3+}(\mathrm{RE}=\mathrm{Er}$ or Tm$)$ are codoped to matrix lattice in the experiments.

Hexagonal $\mathrm{NaYF}_{4}$ host are one of the most effective matrix materials and have been extensively investigated in the UC luminescence properties and applications. Thus, $\beta-\mathrm{NaYF}_{4}: \mathrm{Yb}^{3+}$ / $\left(\mathrm{Tm}^{3+}\right.$ or $\left.\mathrm{Er}^{3+}\right)$ MCs were chosen as a model to achieve the luminescence patterns modulation and the UC red emission enhancement via tuning the excitation positions. Fig. 2 presents excitation position-dependent UC luminescence patterns in the bright field of a single $\beta-\mathrm{NaYF}_{4}: \mathrm{Yb}^{3+} /\left(\mathrm{Tm}^{3+}\right.$ or $\left.\mathrm{Er}^{3+}\right) \mathrm{MC}$ by using a confocal microscopy system. When the NIR excitation laser beam is focused on the center of the single $\beta-\mathrm{NaYF}_{4}: \mathrm{Yb}^{3+} / \mathrm{Tm}^{3+}$ MC particle at a quartz substrate that is mounted on a horizontal 3D moveable platform, a beautiful blue flower-like luminescence pattern is obtained as shown in Fig. $2 \mathrm{a}_{1}$. The strong blue UC light accompanying with red emission is presented in Fig. $2 b_{1}$, which is corresponding to the electronic transitions of ${ }^{1} \mathrm{D}_{2} \rightarrow{ }^{3} \mathrm{~F}_{4}$ (center at $450 \mathrm{~nm}$ ), ${ }^{1} \mathrm{G}_{4} \rightarrow{ }^{3} \mathrm{H}_{6}$ (center at $475 \mathrm{~nm}$ ), ${ }^{1} \mathrm{G}_{4} \rightarrow{ }^{3} \mathrm{~F}_{4}$ (center at $645 \mathrm{~nm}$ ), ${ }^{3} \mathrm{~F}_{2} \rightarrow{ }^{3} \mathrm{H}_{6}$ (center at $695 \mathrm{~nm}$ ) and ${ }^{3} \mathrm{H}_{4} \rightarrow{ }^{3} \mathrm{H}_{6}$ (center at $800 \mathrm{~nm}$ ), respectively. It is easy to observe that the UC red emission intensity is much weaker than the blue emission intensity. However, when the laser beam is transferred to the side of the single $\beta-\mathrm{NaYF}_{4}: \mathrm{Yb}^{3+}$ / $\mathrm{Tm}^{3+} \mathrm{MC}$ particle [Fig. 2a $\mathrm{a}_{2}$, a special UC luminescence phenomenon that the bright directional red emission exhibits 


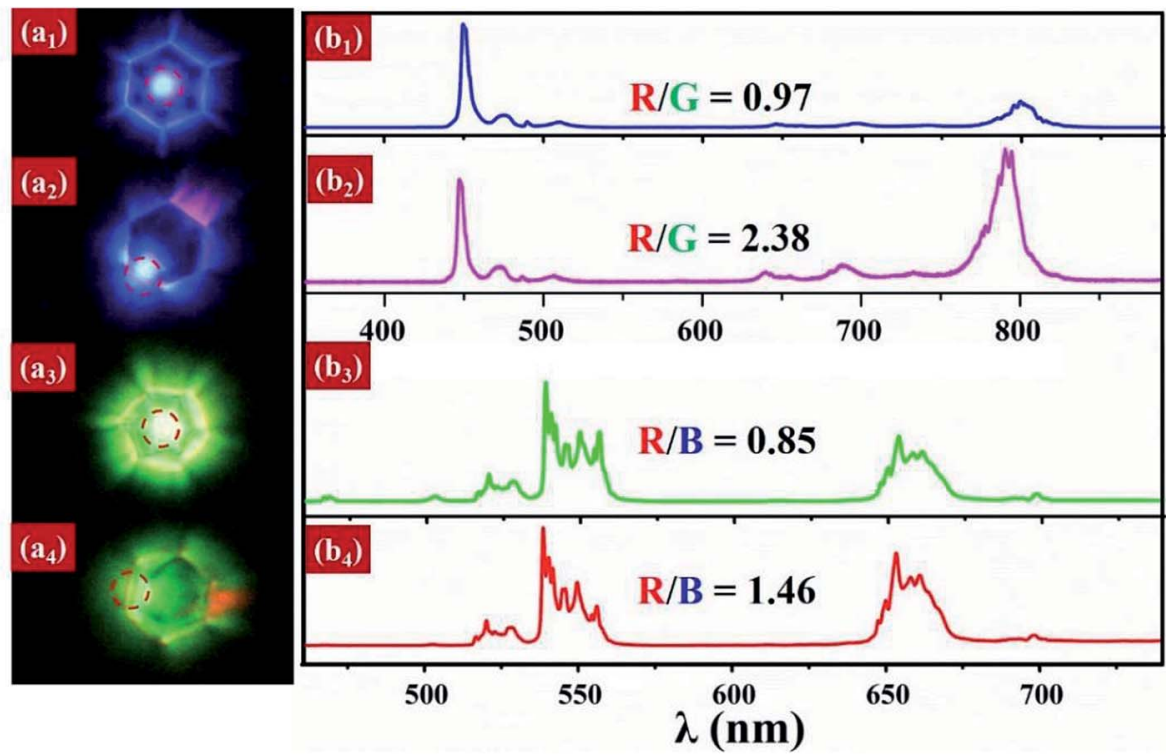

Fig. 2 (a) Schematic of excitation positions and UC luminescence images in the bright field of a single $\beta-\mathrm{NaYF}_{4}: \mathrm{Yb}^{3+} /\left(\mathrm{Tm}^{3+} \mathrm{or} \mathrm{Er}^{3+}\right) \mathrm{MC}_{\mathrm{O}}$ on a quartz substrate under $980 \mathrm{~nm}$ laser excitation. (b) The corresponding UC emission spectra. For clarity, the first two UC emission spectra are normalized to their intensities at $450 \mathrm{~nm}$ from $\beta-\mathrm{NaYF}_{4}: \mathrm{Yb}^{3+} / \mathrm{Tm}^{3+}$, and the last two spectra are normalized to their intensities at $540 \mathrm{~nm}$ from $\beta$ $\mathrm{NaYF}_{4}: \mathrm{Yb}^{3+} / \mathrm{Er}^{3+}$

at one side of the single MP is observed [Fig. $2 \mathrm{~b}_{2}$ ]. It is pointed out that the position of incident laser can be controlled by tuning the moveable platform. The red emission position and the excitation point position of the MC are exactly symmetrical. Furthermore, the red emission intensity is obviously stronger than the blue one. According to their spectra, it is found that the red-to-blue $(\mathrm{R} / \mathrm{B})$ emission intensity ratio increases from 0.97 to 2.38 along with a change of excitation position from the center to the side of MC. These observations suggest that the other luminescence ion doped in the $\mathrm{NaYF}_{4} \mathrm{MC}$ particle might affect their luminescence property under the same measurement condition. Then, when the luminescence center is replaced with $\mathrm{Er}^{3+}$ ions, the special UC emission property of a single MC does not change as shown in Fig. $2 a_{3}, a_{4}$ and $b_{3}, b_{4}$. Obviously, the incident position-dependent emission patterns of the single $\mathrm{NaYF}_{4}: \mathrm{Yb}^{3+} /\left(\mathrm{Tm}^{3+}\right.$ or $\left.\mathrm{Er}^{3+}\right) \mathrm{MC}$ don't rely on a specific luminescence ion. Therefore, these results indicate that the luminescent patterns control and the red emission enhancement for a single MC can be obtained simultaneously by tuning the focal point position of the excitation laser, in which are based on the special structure and configuration of the single $\mathrm{NaYF}_{4}: \mathrm{Yb}^{3+}$ / $\left(\mathrm{Tm}^{3+}\right.$ or $\left.\mathrm{Er}^{3+}\right) \mathrm{MC}$.

To prove the unique luminescence phenomena dependent on the structure and configuration of the single $\mathrm{MC}$, we consider that the especial-shaped $\beta-\mathrm{NaYF}_{4}: \mathrm{Yb}^{3+} / \mathrm{Tm}^{3+} \mathrm{MC}$ is changed for $\beta-\mathrm{NaYF}_{4}: \mathrm{Yb}^{3+} / \mathrm{Tm}^{3+}$ microrod. Based on the same confocal microscopy detection system and measuring condition, the strong UC light outputs like white-blue torch from the endpoint of a single $\beta-\mathrm{NaYF}_{4}: \mathrm{Yb}^{3+} / \mathrm{Tm}^{3+}$ microrod when a laser beam vertically excites in the end or another end of the microrod as shown in the inset of Fig. 3A and C. While the excitation light locates on the middle of the microrod, a beautiful candyshaped luminescence pattern appears in the body of the microrod [Fig. 3B]. Although the observed emission patterns of the single $\beta-\mathrm{NaYF}_{4}: \mathrm{Yb}^{3+} / \mathrm{Tm}^{3+}$ microrod is different for three excitation positions, the UC emission intensities are nearly invariable in three cases, as shown in Fig. 3. These demonstrate that the emission patterns except spectral shape and intensity from single $\beta-\mathrm{NaYF}_{4}: \mathrm{Yb}^{3+} / \mathrm{Tm}^{3+}$ microrod can be controlled via adjusting the position of the focused excitation laser beam.

Therefore, the flower-like luminescence pattern and directional red emission pattern with a red emission enhancement depend on the geometry of the single $\mathrm{NaYF}_{4}: \mathrm{Yb}^{3+} /\left(\mathrm{Tm}^{3+}{\left.\text { or } \mathrm{Er}^{3+}\right)}^{3+}\right.$ MC. Firstly, when the focal point position of the excitation laser

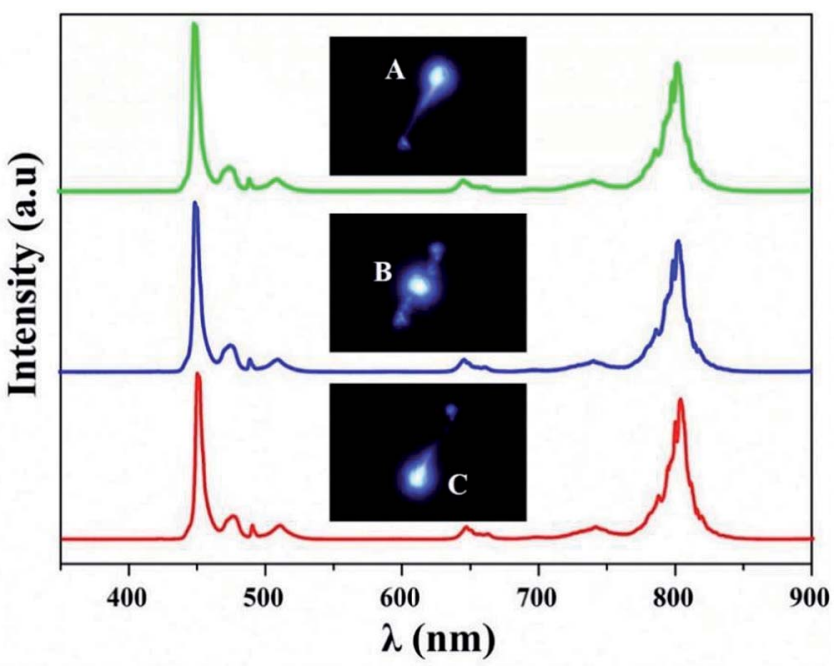

Fig. 3 UC emission spectra and luminescence photos of a single $\beta$ $\mathrm{NaYF}_{4}: \mathrm{Yb}^{3+} / \mathrm{Tm}^{3+}$ microrod with $980 \mathrm{~nm}$ excitation. Note that each spectrum is normalized to its intensity at $450 \mathrm{~nm}$. 


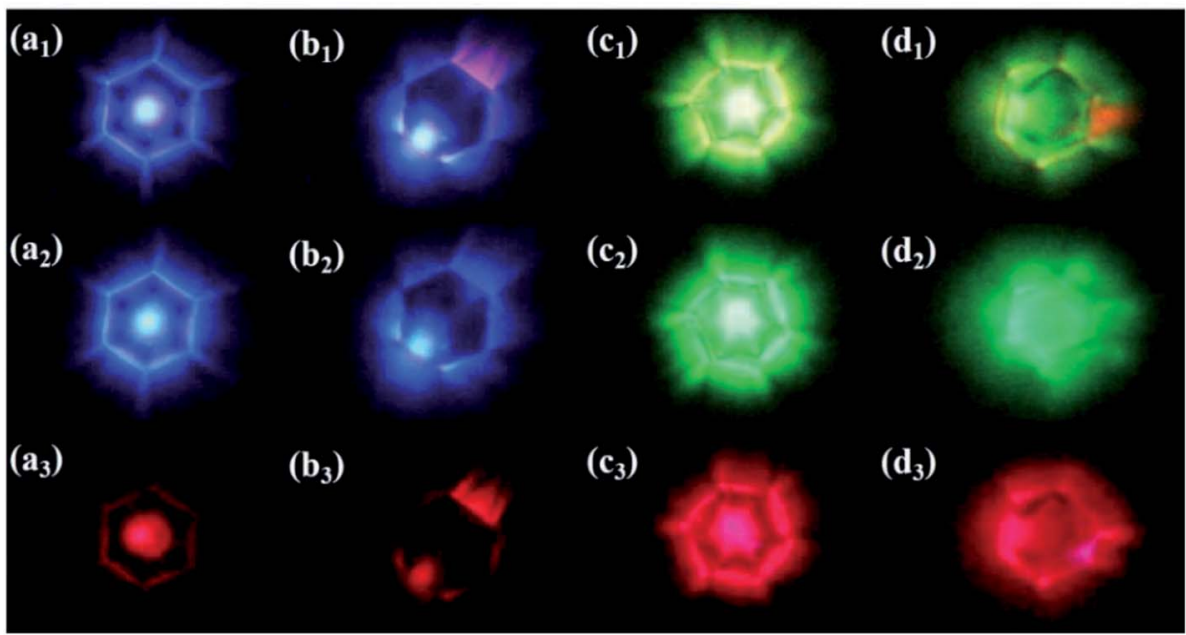

Fig. $4\left(a_{1}, c_{1}\right)$ and $\left(b_{1}, d_{1}\right)$ are the $U C$ emission photographs when the particle is excited at center and side edge from the single $\beta-\mathrm{NaYF}_{4}: \mathrm{Yb}^{3+} /$ $\left(\mathrm{Tm}^{3+}\right.$ or $\left.\mathrm{Er}^{3+}\right) \mathrm{MC}$, respectively. $\left(\mathrm{a}_{2}\right.$ and $\left.\mathrm{b}_{2}\right)$ Blue light emission and $\left(\mathrm{c}_{2}\right.$ and $\left.\mathrm{d}_{2}\right)$ green light emission photographs are obtained via using a low pass filter, $\left(a_{3}\right.$ and $\left.b_{3}\right)$ red light emission and $\left(c_{3}\right.$ and $\left.d_{3}\right)$ red light emission photographs are observed through using a high pass filter.

is on the center of the single MC particle, the red emission doesn't seem existed according to the luminescence photographs of the blue or green flower-like emission pattern as shown in the Fig. $4 \mathrm{a}_{1}$ and $\mathrm{c}_{3}$. But the UC emission spectra present the result that the UC luminescence consists of blue (green) and red light as shown in the Fig. $2 b_{1}$ and $b_{3}$. To further clearly observe the spatial distribution of the UC luminescence, the individual blue (green) and red emission patterns of the single $\mathrm{NaYF}_{4}: \mathrm{Yb}^{3+} /\left(\mathrm{Tm}^{3+}\right.$ or $\left.\mathrm{Er}^{3+}\right)$ MC particle through using filter are exhibited in Fig. $4 a_{2}, a_{3}$ and $c_{2}, c_{3}$, respectively. The optical images show that the blue (green) emission intensity is much larger than the red one. The distribution mechanism of UC luminescence color and intensity is interpreted by the scattering theory. When the NIR laser excites the $\mathrm{NaYF}_{4}: \mathrm{Yb}^{3+}$ / $\left(\mathrm{Tm}^{3+}\right.$ or $\left.\mathrm{Er}^{3+}\right) \mathrm{MC}$, the MC can emit two-color UC luminescence including blue (green) and red light. However, the scatter shortwavelength blue (green) light from the $\mathrm{NaYF}_{4} \mathrm{MC}$ matrix is superior to the scatter long-wavelength red light according to the scattering theory. Therefore, we see the obvious blue (green) luminescence pattern via the camera though the red emission exists. The formation of the beautiful flower-like emission pattern stems from the internal light reflection and waveguide effect. The reflected light and transmitted ray include NIR excitation light and the UC emission light, in which are confined in the host material. Based on the structure characteristic of MC, it is noted that the observed flower-like pattern should be mainly resulted from total internal reflection effect. Hence, the reflected light in the matrix transmits radially to six edge sides of the MC particle forming the observed fascinating flower-like pattern.

In addition, when the focal point position of the excitation laser is adjusted on the side edge of the $\mathrm{NaYF}_{4}: \mathrm{Yb}^{3+} /\left(\mathrm{Tm}^{3+}\right.$ or $\mathrm{Er}^{3+}$ ) MC particle, the luminescence patterns changed and the red emission enhanced relative to the blue (green) one is
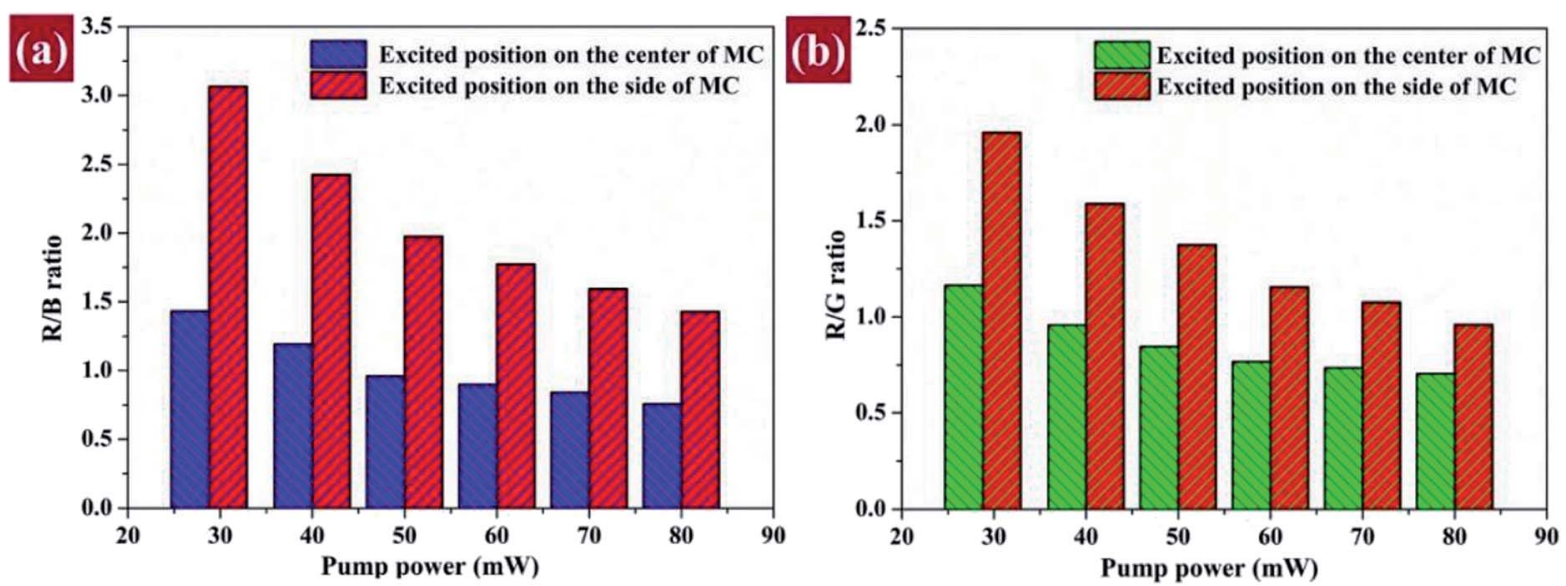

Fig. 5 ( $a$ and $b$ ) The R/B and the R/G emission intensity ratios of the single $\beta-\mathrm{NaYF}_{4}: \mathrm{Yb}^{3+} / \mathrm{Tm}^{3+} \mathrm{MC}$ and $\beta-\mathrm{NaYF}_{4}: \mathrm{Yb}^{3+} / \mathrm{Er}^{3+} \mathrm{MC}$ with the increasing excitation power, respectively. 

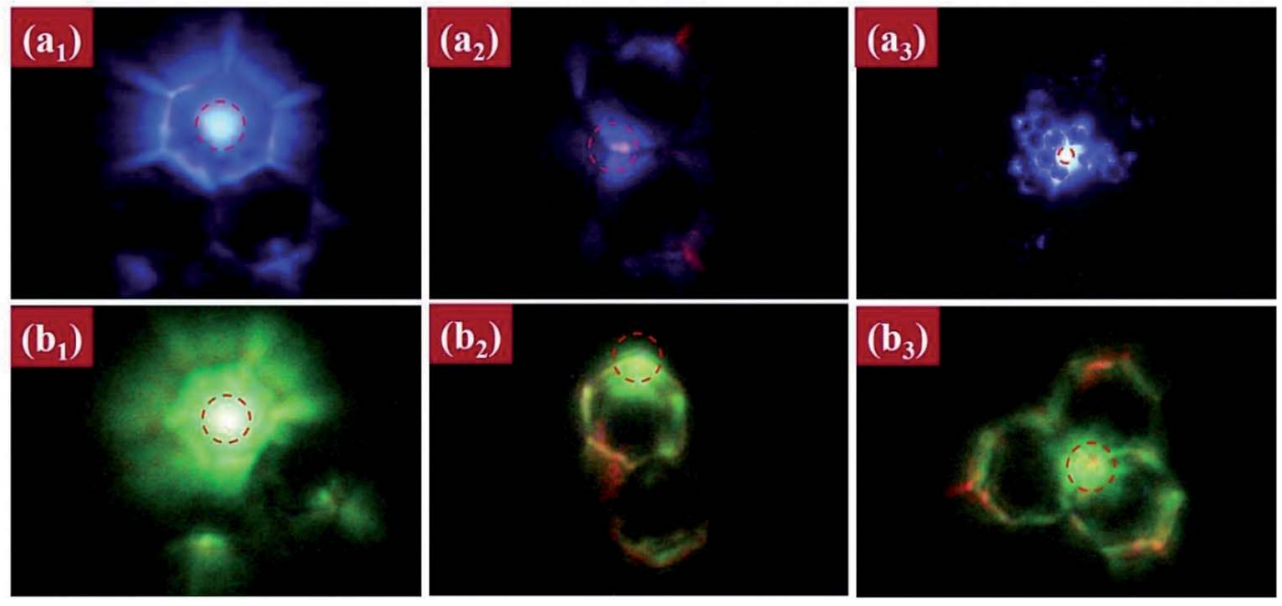

Fig. 6 UC luminescence images of the multiple MCs upon $980 \mathrm{~nm}$ excitation. $\left(a_{1}\right)-\left(a_{3}\right)$ and $\left(b_{1}\right)-\left(b_{3}\right)$ Multiple $\beta-N Y_{F}: Y^{3+} / T m^{3+} M C s$ and $\beta-$ $\mathrm{NaYF}_{4}: \mathrm{Yb}^{3+} / \mathrm{Er}^{3+} \mathrm{MCs}$, respectively. The red circles indicate the position of the excitation.

achieved. Considering the geometry and the light scattering effect of the particle, the spatial distribution of UC luminescence color is different from the first case. As the optical images shown in the Fig. $4 b_{2}, b_{3}$ and $d_{2}, d_{3}$, the blue (green) emission is mainly at the excitation point position and the red emission is at the symmetrical position of the MC particle by using the optical filters. In this case, the confining reflected light and transmitted ray together transmit along the edge lace of the MC particle via total internal reflection, resulting in an increase from the absorption of $\mathrm{Yb}^{3+}$ ions with increasing the optical path of the NIR excitation light in the MC media. In addition, the propagation process of the various rays would not only accompany with scatter effect, but also be incessantly repeated re-absorption and re-emitting processes, this leads to a large increase in the intensities ratio of the red to blue (green), and ultimately, a red flame-like pattern is formed in the side of particle. ${ }^{34}$ This finding reveals that the $\mathrm{NaYF}_{4}: \mathrm{Yb}^{3+} /\left(\mathrm{Tm}^{3+}\right.$ or $\mathrm{Er}^{3+}$ ) MC particle based on the six smooth sides lace could acts as an active optical waveguide and presents a promising characteristic annular resonant microcavity for light propagation.

To further demonstrate the mechanisms of the luminescence patterns variation and red emission enhancement, the excitation power dependences of the UC luminescence intensity of the single $\beta-\mathrm{NaYF}_{4}: \mathrm{Yb}^{3+} /\left(\mathrm{Tm}^{3+}\right.$ or $\left.\mathrm{Er}^{3+}\right) \mathrm{MC}$ particle are separately analyzed for the two excitation positions. As shown in Fig. 5a, the R/B emission intensity ratios from the excitation position on the side of the single $\mathrm{NaYF}_{4}: \mathrm{Yb}^{3+} / \mathrm{Tm}^{3+} \mathrm{MC}$ are much greater than the R/B ratios from the excitation position on the center of the particle with increase of the pump power. The R/G intensity ratios of the single $\mathrm{NaYF}_{4}: \mathrm{Yb}^{3+} / \mathrm{Er}^{3+} \mathrm{MC}$ particle also present the similar results exhibited in Fig. 5b. These indicate that the excitation approach based on the waveguide effect could not obtain equivalent UC luminescence intensity with varying excitation power. However, the modulation of UC luminescence patterns and the enhancement of red light is still achieved by the confining propagation of light along the six sides lace around the MC particle and the redistributing intensity of light in space via the waveguiding.

In fact, the luminescence patterns and intensities could be efficiently controlled by tuning the focal position of the excitation laser, in which is based on the optical waveguide effect. The optical effect is also adapted to the multiple $\beta$ $\mathrm{NaYF}_{4}: \mathrm{Yb}^{3+} /\left(\mathrm{Tm}^{3+}\right.$ or $\left.\mathrm{Er}^{3+}\right)$ MCs. As shown in Fig. 6, the UC emission light can be propagated from the excitation point position to the symmetrical position of the excitation point along the sides lace of the MCs, and then transmitted from one MC particle to the other neighbored ones through the connecting edges in the system of multiple MCs, forming a lot of beautiful luminescence patterns. These findings can be widely used in anti-counterfeiting and optical barcoding based on a single MC system or three-dimensional color display.

\section{Conclusions}

In summary, we report some interesting phenomena that the UC luminescence patterns could be effectively controlled and the red emission intensities could be enhanced from a single $\beta$ $\mathrm{NaYF}_{4}: \mathrm{Yb}^{3+} /\left(\mathrm{Tm}^{3+}\right.$ or $\left.\mathrm{Er}^{3+}\right) \mathrm{MC}$ via tuning the excitation mode. When the focal point position of the excitation NIR laser is adjusted from the center to one side lace of the MC particle, the UC luminescence pattern would be transformed from blue or green flower-like emission pattern to the red flame-like irradiation pattern accompanied with a red luminescence enhancement. However, the luminescence properties are irrelevant to a specific luminescence center, they are sensitive to the crystal structure, excited position, pump power, and numbers. Their corresponding physical mechanism is explained by the optical waveguide effect based on the total reflection effect. We believe that the unique-structure micro/nano-crystal materials and excited mode would open a promising application in threedimensional color display, optical waveguides and optical communication. 


\section{Conflicts of interest}

There are no conflicts to declare.

\section{Acknowledgements}

This work is supported by the National Science Foundation of China (11604262), Shaanxi Provincial Research Plan for Young Scientific and Technological New Stars (Program No. 2015KJXX40), the New Star Team of Xi'an University of Posts \& Telecommunications, the Natural Science Basic Research Plan in Shaanxi Province of China (Grant No. 2018JM1052, 2018JQ1070), Natural Science Foundation of Shaanxi Provincial Department of Education (Grant No. 18JK0712), and the Open Research Fund of Key Laboratory of Spectral Imaging Technology, Chinese Academy of Sciences (LSIT201810D).

\section{References}

1 B. Zhou, B. Shi, D. Jin and X. Liu, Nat. Nanotechnol., 2015, 10, 924.

2 S. Wen, J. Zhou, K. Zheng, A. Bednarkiewicz, X. Liu and D. Jin, Nat. Commun., 2018, 9, 2415.

3 Y. Hao, S. Lv, Z. Ma and J. Qiu, RSC Adv., 2018, 8, 1216512172.

4 L. D. Sun, H. Dong, P. Z. Zhang and C. H. Yan, Annu. Rev. Phys. Chem., 2015, 66, 619-642.

5 Z. Yin, H. Li, W. Xu, S. Cui, D. Zhou, X. Chen, Y. Zhu, G. Qin and H. Song, Adv. Mater., 2016, 28, 2518-2525.

6 D. Yang, Z. Hou, Z. Cheng, C. Li and J. Lin, Chem. Soc. Rev., 2015, 44, 1416-1448.

7 S. T. Dibaba, R. Y. Wei, W. S. Xi, L. Zhao, L. Y. Shi, W. Ren, T. Mayrd and L. N. Sun, RSC Adv., 2018, 8, 35706-35718.

8 M. Yang, J. Ren and R. Zhang, J. Alloys Compd., 2017, 714, 160-167.

9 T. Dilbeck, S. P. Hill and K. Hanson, J. Mater. Chem. A, 2017, 5, 11652-11660.

10 M. Luoshan, L. Bai, C. Bu, X. Liu, Y. Zhu, K. Guo, R. Jiang, M. Li and X. Zhao, J. Power Sources, 2016, 307, 468-473.

11 W. Zheng, P. Huang, D. Tu, E. Ma, H. Zhu and X. Chen, Chem. Soc. Rev., 2015, 44, 1379-1415.

12 P. Zhao, Y. Zhu, X. Yang, X. Jiang, J. Shen and C. Li, J. Mater. Chem. A, 2014, 2, 16523-16530.

13 R. Deng, F. Qin, R. Chen, W. Huang, M. Hong and X. Liu, Nat. Nanotechnol., 2015, 10, 237.
14 X. Zhu, Q. Su, W. Feng and F. Li, Chem. Soc. Rev., 2017, 46, 1025-1039.

15 D. Chen, M. Xu and P. Huang, Sens. Actuators, B, 2016, 231, 576-583.

16 Y. Liu, Y. Lu, X. Yang, X. Zheng, S. Wen, F. Wang, X. Vidal, J. Zhao, D. Liu and Z. Zhou, Nature, 2017, 543, 229.

17 B. Yang, Y. Wang, T. Wei, Y. Pan, E. Zhou, Z. Yuan, Y. Han, M. Li, X. Ling and L. Yin, Adv. Funct. Mater., 2018, 1801782.

18 L. Tian, Y. Shang, S. Hao, Q. Han, T. Chen, W. Lv and C. Yang, Adv. Funct. Mater., 2018, 28, 1803946.

19 W. You, D. Tu, W. Zheng, X. Shang, X. Song, S. Zhou, Y. Liu, R. Li and X. Chen, Nanoscale, 2018, 10, 11477-11484.

20 C. Homann, L. Krukewitt, F. Frenzel, B. Grauel, C. Würth, U. Resch-Genger and M. Haase, Angew. Chem., Int. Ed., 2018, 57, 8765-8769.

21 L. Chen, Y. Rong, M. Ren, W. Wu, M. Qin, C. Pan, Q. Ma, S. Liu, B. Wu, E. Wu, J. Xu and H. Zeng, J. Phys. Chem. C, 2018, 122, 15666-15672.

22 J. Dong, Z. Zhang, H. Zheng and M. Sun, Nanophotonics, 2015, 4, 472-490.

23 S. Schietinger, L. d. S. Menezes, B. r. Lauritzen and O. Benson, Nano Lett., 2009, 9, 2477-2481.

24 B. Chen, W. Kong, Y. Liu, Y. Lu, M. Li, X. Qiao, X. Fan and F. Wang, Angew. Chem., Int. Ed., 2017, 56, 10383-10387.

25 L. Yan, B. Zhou, N. Song, X. Liu, J. Huang, T. Wang, L. Tao and Q. Zhang, Nanoscale, 2018, 10, 17949-17957.

26 T. Wang, H. Yu, C. K. Siu, J. Qiu, X. Xu and S. F. Yu, ACS Photonics, 2017, 4, 1539-1543.

27 C. X. Li, Z. W. Quan, J. Yang, P. P. Yang and J. Lin, Inorg. Chem., 2007, 46, 6329-6337.

28 A. Nadort, J. B. Zhao and E. M. Goldys, Nanoscale, 2016, 8, 13099-13130.

29 M. Y. Ding, C. H. Lu, Li. H. Cao, Y. R. Ni and Z. Z. Xu, CrystEngComm, 2013, 15, 8366-8373.

30 M. Law, D. J. Sirbuly, J. C. Johnson, J. Goldberger, R. J. Saykally and P. Yang, Science, 2004, 305, 1269-1273.

31 Y. Zhang, L. Zhang, R. Deng, J. Tian, Y. Zong, D. Jin and X. Liu, J. Am. Chem. Soc., 2014, 136, 4893-4896.

32 Q. Han, C. Zhang, C. Wang, Z. Wang, C. Li, W. Gao, J. Dong, E. He, Z. Zhang and H. Zheng, Sci. Rep., 2017, 7, 5371.

33 W. Gao, H. Zheng, Q. Han, E. He and R. Wang, CrystEngComm, 2014, 16, 6697-6706.

34 J. Xu, X. Zhuang, P. Guo, Q. Zhang, W. Huang, Q. Wan, W. Hu, X. Wang, X. Zhu and C. Fan, Nano Lett., 2012, 12, 5003-5007. 